Pierre Pollecot ${ }^{1}$

Bechir DJIMET ${ }^{2}$

Takene NGUI ${ }^{2}$

\section{${ }^{1}$ Cirad}

Département Environnements et Sociétés

Unité de recherche Animal

et gestion intégrée des risques

TA C/22 E, Campus international

de Baillarguet

34398 Montpellier Cedex 5

France

2 Projet CURESS

Parc national de Zakouma

BP 552, N'Djamena

Tchad

\title{
La population
d'éléphants du parc
national de Zakouma \\ La population
d'éléphants du parc
national de Zakouma \\ La population
d'éléphants du parc
national de Zakouma (Tchad)
}

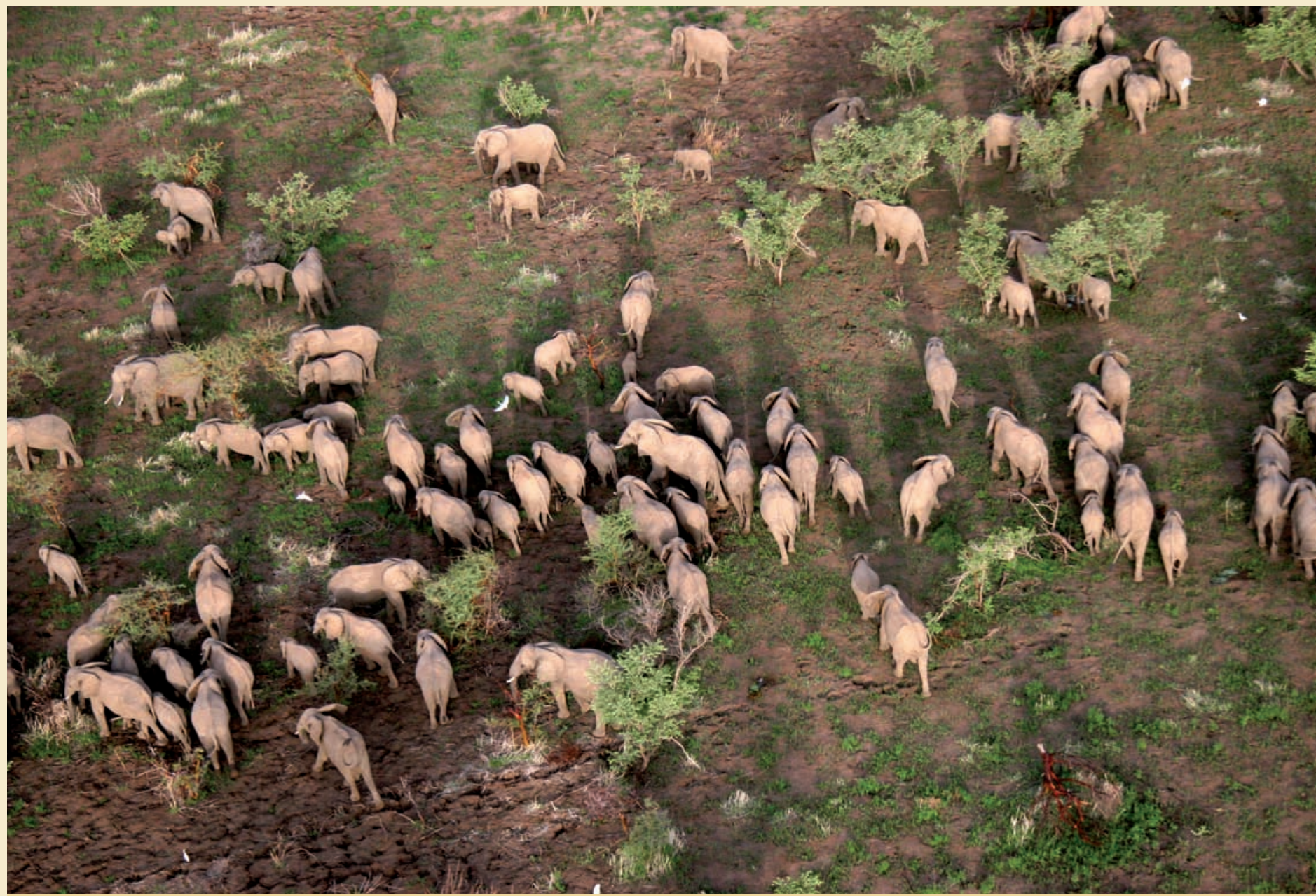




\section{RÉSUMÉ}

\section{LA POPULATION D’ÉLÉPHANTS DU PARC NATIONAL DE ZAKOUMA (TCHAD)}

Dans les années 1970-1980, la faune du parc national de Zakouma, dans le sud-est du Tchad, avait souffert de périodes instables sur le plan politique, de sécheresses récurrentes et d'une sérieuse épidémie de peste bovine. Les mesures de conservation mises en œuvre à partir de 1989, avec le soutien de l'Union européenne, avaient permis une rapide reconstitution de la plupart des espèces de grands mammifères. Les résultats obtenus à la suite de récents dénombrements aériens, réalisés entre 2005 et 2009 , ont cependant révélé une nette diminution de la population d'éléphants. En effet, estimée à environ 3900 individus en 2005, elle a commencé à décliner à partir de la saison sèche 2006 où elle ne comptait plus que 3000 animaux. Les effectifs ont ensuite brutalement régressé de 940 individus en 2008 à seulement 620 en 2009. Cette chute drastique, de près de $70 \%$ de la population d'éléphants, est corrélée à un nombre important de carcasses, correspondant à des éléphants récemment abattus, principalement distribuées dans la partie est de l'aire protégée, au sein des vallées du réseau hydrographique majeur. Le braconnage, qui a toujours constitué une menace pour les éléphants de Zakouma, n'avait jamais atteint une telle intensité et correspond à la reprise du commerce international de l'ivoire en 2006.

Mots-clés : grands mammifères, évolution effectifs de l'éléphant, Zakouma, Tchad.

\section{ABSTRACT}

\section{THE ELEPHANT POPULATION IN ZAKOUMA NATIONAL PARK - CHAD}

In the 1970s and 1980s, the wildlife in Zakouma National Park, in southeastern Chad, had suffered from periods of political instability, recurrent drought and a serious epidemic of rinderpest. The conservation measures implemented as from 1989, with support from the European Union, soon restored most of the large mammal species. However, the results of recent aerial counts carried out from 2005 to 2009 reveal a steep decline in the elephant population. From an estimated total of about 3900 individuals in 2005, the population began to decline in the 2006 dry season, when only 3000 animals were counted. Their numbers then dropped steeply to 940 individuals in 2008 and only 620 in 2009. This dramatic decline, amounting to almost $70 \%$ of the elephant population, is correlated with a large number of carcasses of recently shot elephants, which have been found mainly in the eastern part of the protected areas in the valleys of a large hydrographic network. Poaching, which has always been a threat to the Zakouma elephants, had never before reached such proportions and coincides with the reopening of the international ivory trade in 2006.

Keywords: large mammals, trends in elephant numbers, Zakouma, Chad.

\section{RESUMEN}

\section{LA POBLACIÓN DE ELEFANTES DEL PARQUE NACIONAL DE ZAKOUMA - CHAD}

Durante los años 70 y 80 , la fauna del Parque Nacional de Zakouma, en el sudeste de Chad, sufrió las consecuencias de períodos de inestabilidad política, padeció sequías repetidas y una seria epidemia de peste bovina. Las medidas de conservación aplicadas desde 1980, con el apoyo de la Unión Europea, permitieron una rápida reconstitución de la mayoría de especies de grandes mamíferos. Sin embargo, los resultados que arrojan los recientes censos aéreos, realizados entre 2005 y 2009 , revelan una clara disminución de la población de elefantes. Dicha población estaba estimada en torno a 3900 individuos en 2005 y empezó a decaer a partir de la estación seca de 2006, en la que ya sólo se contaban 3000 animales. A continuación, el número de animales se desplomó brutalmente pasando a 940 individuos en 2008 y a sólo 620 en 2009. Esta drástica caída, de casi el $70 \%$ de la población de elefantes, se pone en correlación con un número importante de cadáveres, correspondientes a animales matados recientemente, localizados básicamente en la zona oriental del área protegida, en los valles de la principal red hidrográfica. El furtivismo, que siempre ha supuesto una amenaza para los elefantes de Zakouma, nunca había alcanzado tal intensidad y se corresponde con la reanudación del comercio internacional de marfil en 2006.

Palabras clave: grandes mamíferos, evolución del número de elefantes, Zakouma, Chad. 


\section{Introduction}

Comme la plupart des aires protégées d'Afrique francophone, le parc national de Zakouma trouve ses origines dans le passé colonial du Tchad. Ce pays abritait alors une faune d'une exceptionnelle richesse, en particulier de grands mammifères. La forte pression de chasse qui prévalait avait conduit de nombreuses espèces au bord de l'extinction, comme le rhinocéros noir, l'éléphant et la girafe. En 1958, le colonel Michel Anna, alors responsable du secteur des chasses, proposa la création de la réserve de faune de Zakouma dans le sud-est du pays. Après l'Indépendance acquise par le Tchad en 1960, et considérant le succès obtenu par cette réserve en termes de reconstitution des effectifs des populations animales, le gouvernement tchadien décida de renforcer la protection de Zakouma en créant le parc national de Zakouma en 1963. Dans les années 1970-1980, le parc a souffert d'une longue période de conflits armés, de sécheresses récurrentes et d'un sévère épisode de peste bovine. Une mission d'évaluation du capital faunique du parc de Zakouma, conduite en 1986, a cependant conclu à la présence de la plupart des espèces de grands mammifères de la zone soudanienne, bien que certaines avec des effectifs réduits (Pfeffer, Thomassey, 1986). Ce rapport encourageant a incité le gouvernement tchadien à demander une assistance internationale pour réhabiliter l'aire protégée. C'est ainsi que l'Union européenne, au travers de divers projets de développement mis en œuvre à partir de 1989 , a contribué à une spectaculaire reconstruction des populations animales de Zakouma et à la sauvegarde de ce patrimoine. Le parc de Zakouma, qui couvre $3000 \mathrm{~km}^{2}$ au sein des savanes soudano-sahéliennes (figure 1), peut être actuellement considéré comme un véritable sanctuaire pour la faune soudanienne de ce pays (ArRANZ et al., 2007).

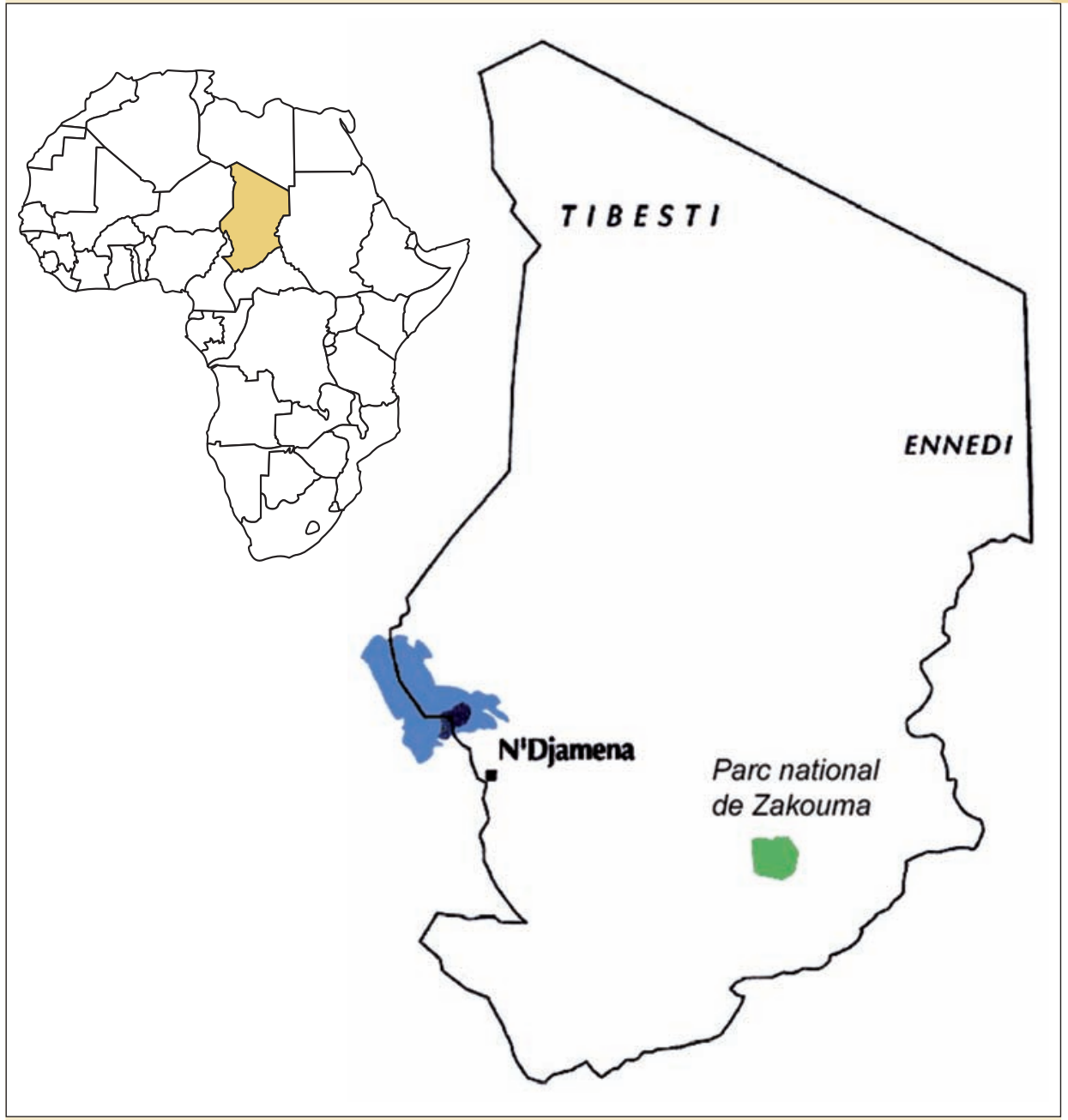

Figure 1.

Situation du parc national de Zakouma au Tchad.

\section{Le constat}

La végétation du parc national de Zakouma est caractérisée par des savanes à Acacia seyal sur les sols hydomorphes et des savanes à combrétacées sur les terrains mieux drainés. De vastes plaines herbeuses, marécageuses en saison des pluies, assurent des ressources en pâturage et en eau à une faune très variée au cours de la saison sèche. Cette aire protégée est riche de soixante-cinq espèces de mammifères et de plus de 370 espèces d'oiseaux. La présence d'espèces emblématiques, éléphant (photo 1) et guépard, la forte densité des grands herbivores et l'importante diversité de grands et petits carnivores font de ce parc un site exceptionnel au sein de l'Afrique francophone.
La figure 2 illustre l'évolution des effectifs des grands mammifères entre 1986 et 2006. Les dénombrements des grands mammifères ont été réalisés, jusqu'en 2002, selon la méthode du comptage aérien par échantillonnage systématique puis par des comptages aériens totaux en 2005 et 2006. Les effectifs des principales espèces chutent à partir de 2005, à l'exception du buffle et du damalisque (Fay et al., 2005). En revanche, les populations augmentent à la suite du comptage de 2006, excepté pour l'éléphant, qui accuse une nette diminution, et du damalisque dont la population demeure relativement stable entre 2002 et 2006. 


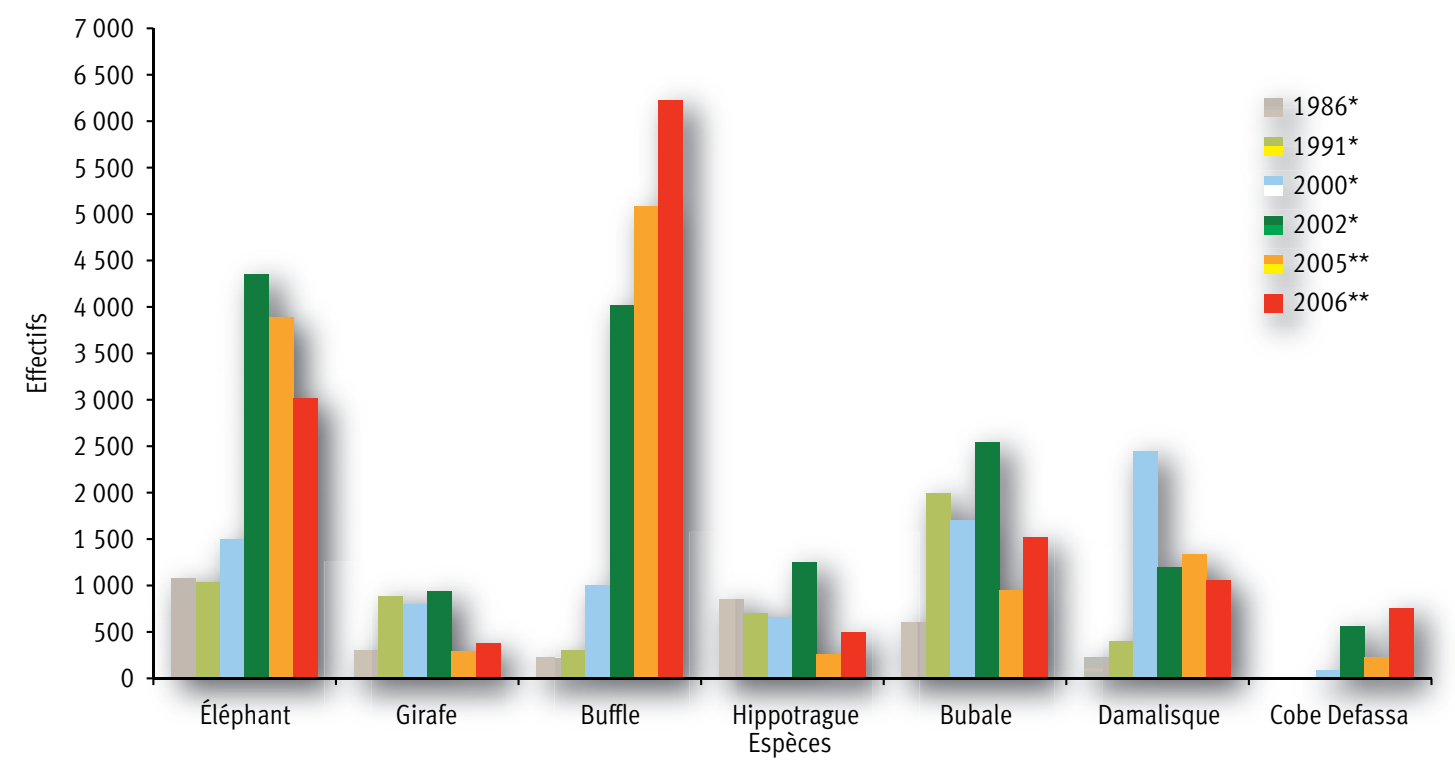

Figure 2.

Évolution des effectifs des principaux grands mammifères du parc national de Zakouma entre 1986 et 2006.

* Comptage aérien par échantillonnage systématique. ** Comptage aérien total.

(Sources : Bousquet, 1986, 1991 ; DejACE et al., 2000 ; MACKIE, 2002 ; FAY et al., 2005, 2006).

Les résultats du dénombrement de 2002 sont des estimations avec, très souvent, un fort intervalle de confiance. Les effectifs obtenus à la suite des comptages totaux sont certainement plus proches de la réalité. L'amorce du déclin de la population d'éléphants à partir de 2002, estimée à 4350 individus (intervalle de confiance : 41,4\%), n'est pas alors alarmante. Le comptage total de 2005 avait conduit au recensement de près de 3900 animaux dont un troupeau de plus de 1000 individus (photo 2).

La densité de 1,3 éléphant par kilomètre carré pouvait être alors considérée comme unique dans une aire protégée d'Afrique francophone.

Le dénombrement réalisé en avril 2006, au cours de la saison sèche, confirme la diminution des effectifs de l'éléphant, avec une population d'environ 3000 individus, soit une perte de près de $22 \%$ en comparaison de 2005 . Ce comptage a été complété par un dénombrement en août, au sein de l'aire protégée et dans la zone périphérique. Ce premier recensement aérien de saison des pluies (total dans le parc et par échantillonnage systématique dans la périphérie de l'aire protégée) a permis de comptabiliser 1500 éléphants dans les limites du parc et 1600 dans les savanes à combrétacées de la zone périphérique (FAY et al., 2006 a et b). Un troupeau estimé à près de 1000 individus fut observé à la limite extérieure sud-ouest du parc dans un peuplement de bambous (Oxytenanthera abyssinica (A. Rich.) Munro) (photo 2).

Les éléphants du parc national de Zakouma effectuent des migrations annuelles du fait d'inondations qui couvrent une partie de l'aire protégée, en particulier l'est du parc, au cours de la saison des pluies (juin-octobre). Ces déplacements saisonniers, décrits pour la première fois par DEPIERRE en 1967, ont été confirmés à la suite d'un suivi télémétrique de plusieurs individus, qui avait conduit à cartographier la mobilité des animaux équipés de balises Argos (DolmiA 2004 ; DolmiA et al., 2007). Les résultats du recensement d'août 2006 ont confirmé ceux obtenus par N. Dolmia et permettent une estimation plus fine de la part de la population émigrante (FAY et al., 2006b.). Au total, 3100 éléphants furent donc comptés en saison des pluies, donnant une population proche de celle recensée au cours de la saison sèche à l'intérieur du parc (3 000 animaux). Ce survol a montré qu'une proportion importante de la population d'éléphants, environ la moitié, quittait l'aire protégée au cours de la saison des pluies. L'observation des troupeaux et des traces fraîches situait les animaux jusqu'à 80 à 100 km des limites du parc, dans le nord en direction de la réserve de faune d'Abou Telfan et dans le sudouest aux abords de la réserve de faune de Siniaka-Minia.

Un comptage aérien par échantillonnage systématique réalisé en avril-mai 2008 , dans le cadre des activités de suivi des populations de grands mammifères du parc de Zakouma, a mis en évidence une chute drastique de la population d'éléphants par rapport au précédent dénombrement de 2006. Celle-ci fut estimée à seulement 937 individus (intervalle de confiance : 115,5\%) à partir de 187 individus observés (PoILECOT, 2008). Seuls trois troupeaux furent enregistrés comprenant 21, 24 et 142 individus. Aucun éléphant ne fut repéré lors des vols complémentaires permettant de rejoindre les points de départ des transects ou le camp de base à la fin des comptages, ce qui est exceptionnel pour le parc de Zakouma. Enfin, en 2009, un comptage total de saison sèche a de nouveau confirmé le déclin de la population d'éléphants du parc avec une estimation ne dépassant pas 620 individus (Potgieter et al., 2009). 


\section{Un nombre croissant de carcasses}

À la diminution des effectifs de la population d'éléphants correspond une augmentation croissante des cadavres d'éléphants observés. Les carcasses d'éléphants ont été classées en trois catégories relatives à leur état (photo 3) :

- "fraîche ", carcasse inférieure à trois semaines: carcasse intacte, présence de vautours et/ou de déjections blanches (crottes de vautours) bien visibles, végétation piétinée et auréole humide bien marquée autour de la carcasse ;

- "récente ", carcasse de trois semaines à un an : peau encore présente, squelette encore partiellement articulé, absence de crottes de vautours et auréole disparue ;

- « vieille », carcasse supérieure à un an : carcasse réduite à des os blanchis et dispersés.

Dans le parc de Zakouma, sous un climat semi-aride, la dégradation des carcasses s'avère rapide du fait des inondations et des feux de brousse annuels.

Le tableau I et la figure 3 synthétisent les données des carcasses, pour chaque catégorie, collectées au cours des dénombrements effectués entre 2002 et 2009 . Au sein du parc, le nombre de carcasses fraîches est négligeable à partir du comptage de 2005. Celui des carcasses récentes, important en 2002, régresse ensuite pour s'ac-

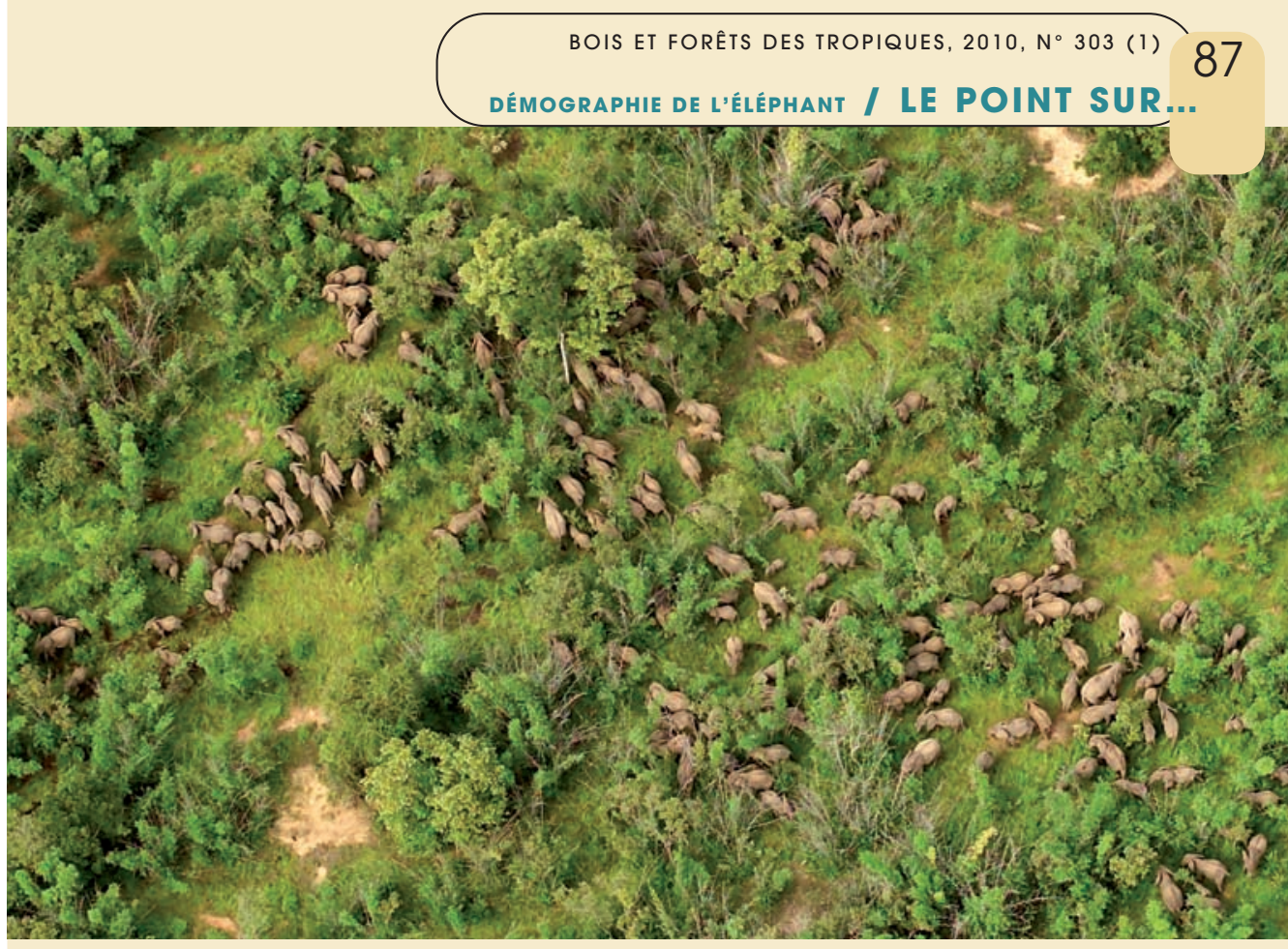

Photo 2.

Important troupeau d'éléphants dans un peuplement de bambou.

Photo M. Fay.

croître de nouveau à partir de la saison des pluies 2006 et atteindre sa plus forte valeur en 2008 avec 124 cadavres effectivement observés (photo 4), conduisant à une estimation de 619 carcasses récentes (intervalle de confiance : 56,2\%) à l'échelle de l'aire protégée, après l'analyse des données. Au cours de la saison des pluies 2006, l'effectif des vieilles carcasses régresse du fait de la densité de la végétation qui limite la visibilité et donc la détection des squelettes. En 2007, un survol exploratoire complémentaire dans le nord de la zone périphérique a de nouveau conduit au recensement de nouvelles carcasses (FAY, 2007). Le comptage total effectué au cours de la saison sèche 2009 a enregistré 11 carcasses récentes supplémentaires. Les deux dénombrements de 2006, en particulier celui de saison des pluies, ont révélé des activités de braconnage, avec une forte proportion de carcasses fraîches (44) et récentes (63), qui n'étaient pas jusqu'alors soupçonnées, tant dans le parc que dans la zone périphérique, et qui se sont poursuivies.

Le comptage des vieilles carcasses inclut ainsi des squelettes vieux de un à trois ans. Les 369 squelettes comptés en 2008 donnent une estimation de 1666 animaux abattus (intervalle de confiance : $43 \%$ ). L'effectif de 770 vieilles carcasses, réellement observé en 2009 à la suite du comptage total, est également très élevé.

Tableau I.

Effectifs des carcasses d'éléphants (estimés ou comptés) dans le parc de Zakouma au cours de la période 2002-2009.

\begin{tabular}{|c|c|c|c|c|c|c|c|}
\hline Classe & Avril 2002* & Avril $2005^{\star *}$ & Avril 2006** & & 2006 & Avril-mai 2008* & Mars 2009** \\
\hline & Parc & Parc & Parc & Parc** & Périphérie* & Parc & Parc \\
\hline Fraîche & $22(4)$ & 2 & 0 & 6 & 38 & 0 & 0 \\
\hline Récente & $38(7)$ & 0 & 3 & 34 & 29 & 619 (124) & 11 \\
\hline Vieille & $108(18)$ & 177 & 156 & 39 & 32 & 1666 (369) & 770 \\
\hline Total & 168 & 179 & 159 & 79 & 99 & 2285 & 781 \\
\hline
\end{tabular}

* Comptage aérien par échantillonnage systématique.

** Comptage aérien total.

Chiffres entre parenthèses : effectifs observés. 


\section{Un changement dans la distribution des carcasses}
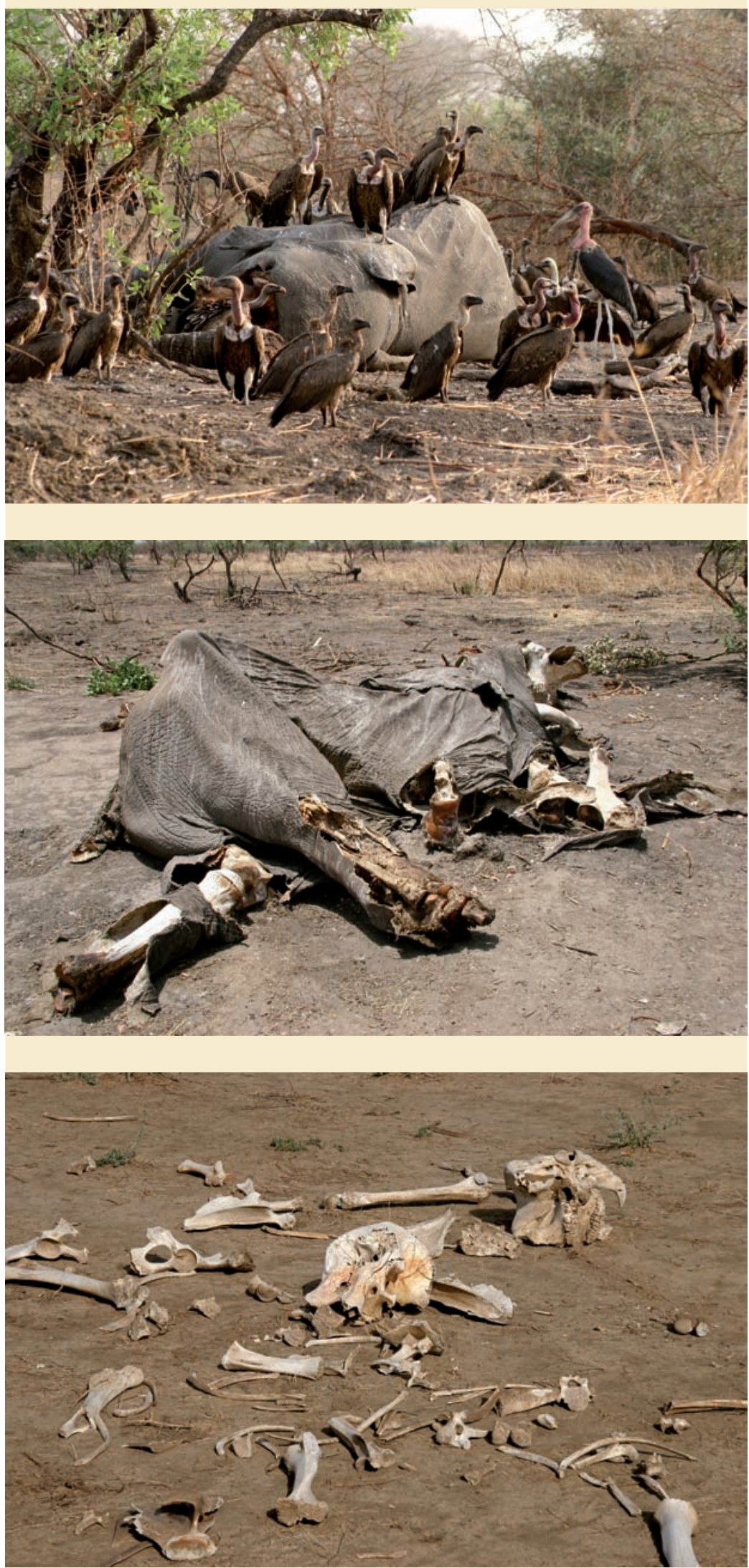

Photo 3.

Classes d'âge relatives aux carcasses d'éléphants.

De haut en bas : carcasses fraîche,

récente,

vieille.

Photos P. Poilecot.
Jusqu'en 2005, la distribution des carcasses, toutes catégories confondues, se limitait principalement à la partie ouest du parc où les patrouilles de surveillance sont moins nombreuses du fait d'un réseau de pistes limité et donc d'un accès difficile (FAY et al., 2006 a). À partir de 2006, la cartographie des observations concentre davantage les squelettes dans la partie est du parc.

La figure 4 illustre la distribution des vieilles carcasses à la suite du dénombrement aérien d'avril-mai 2008. Bien que ces carcasses soient réparties dans l'ensemble de l'aire protégée, la partie occidentale en abrite un plus grand nombre, localisé dans les vallées des principaux cours d'eau. La distribution des carcasses récentes (figure 5) concerne la partie centrale de cette région du parc.

Les derniers dénombrements montrent donc une distribution des carcasses qui s'étend à l'ensemble du parc avec, cependant, une zone privilégiée correspondant au réseau hydrographique principal constitué du Bahr Salamat, qui demeure permanent pendant la saison sèche, et de ses principaux affluents assurant des ressources en eau pendant une partie de la saison défavorable seulement. Également, le comptage de 2008 met en évidence un nombre important de carcasses dans les environs du poste principal des gardes du parc, Zakouma, et du campement touristique de Tinga. 


\section{Pour conclure : inquiétudes}

Le Tchad abrite une riche biodiversité mais qui demeure encore mal connue. Le réseau de parcs nationaux et réserves, qui couvre 10,2\% du territoire, est représentatif de la diversité des écosystèmes de cette partie de l'Afrique centrale. Cependant, les sécheresses récurrentes et les activités anthropiques ont altéré de nombreux habitats naturels et fragmenté l'aire de distribution de l'éléphant qui couvre actuellement environ $260000 \mathrm{~km}^{2}$, soit $21 \%$ de la superficie du pays. La population d'éléphants, comprise entre 3500 et 6000 animaux, est divisée en huit "sous-populations" depuis le lac Fitri au nord, dans le domaine sahélien, jusque dans le domaine soudanien au sud.

Cette valeur ne représente pas une estimation nationale du fait de données imprécises, de mouvements de troupeaux entre le Cameroun et le Tchad et de zones n'ayant jamais été explorées (DoLMIA, 2004 ; IUCN/Ssc, 2007).

Peu de données permettent de savoir s'il existe ou non des connexions entre ces différentes populations. TCHAMBA et al. (1997) ont rapporté que 300 à 400 éléphants effectuent des mouvements entre la région du lac Tchad et le Nord-Cameroun mais qu'ils séjournent plus longtemps dans ce dernier. Cette observation est corroborée par Loomis (2002), qui précise que les éléphants en provenance de la savane du Nord-Cameroun se dispersent vers le Nigeria et le Tchad. DEJACE (1996) a avancé que des troupeaux migraient entre le sud du Tchad et le nord de la République centrafricaine ; mais ces informations n’ont jamais été vérifiées et semblent peu vraisemblables du fait d'inondations couvrant de vastes superficies en saison des pluies et de la présence de nombreux éleveurs transhumants à la fin de l'hivernage. En revanche, les suivis télémétriques ont confirmé des voies migratoires des éléphants entre le parc de Zakouma et les réserves de faune de Siniaka-Minia et d'Abou Telfan situés respectivement au sud-ouest et au nord-ouest.

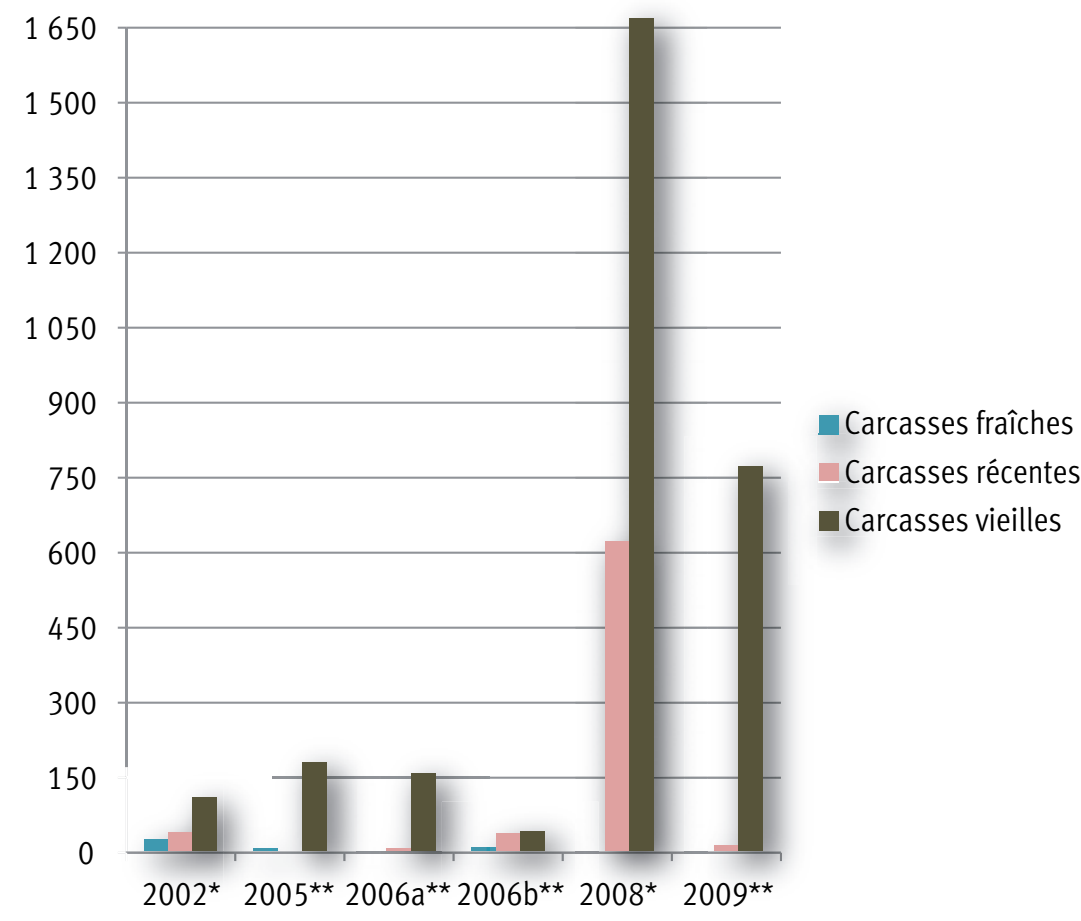

Figure 3.

Évolution des effectifs des carcasses "fraîches », "récentes » et "vieilles » au cours de la période 2002-2009.

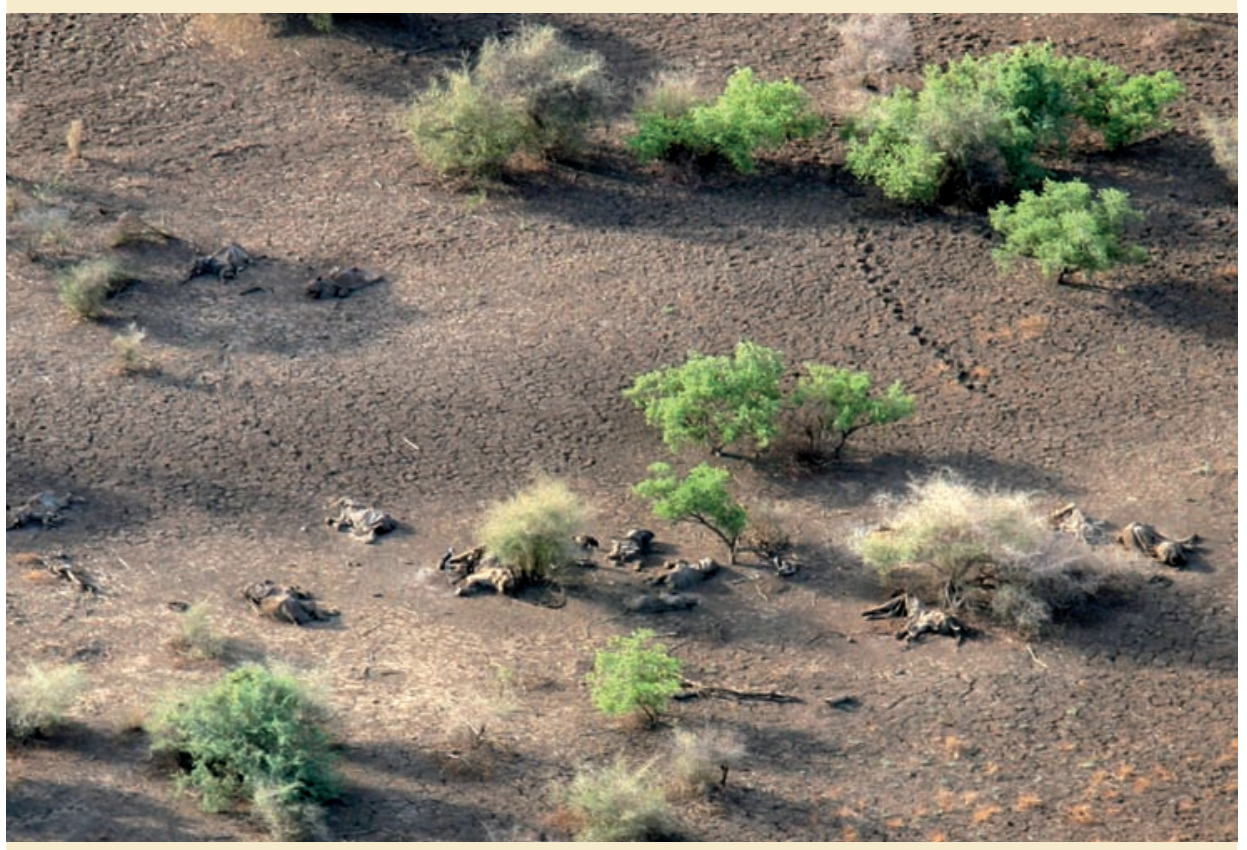

Photo 4.

Groupe d'éléphants massacrés dans une savane arbustive claire à combrétacées.

Photo P. Poilecot. 


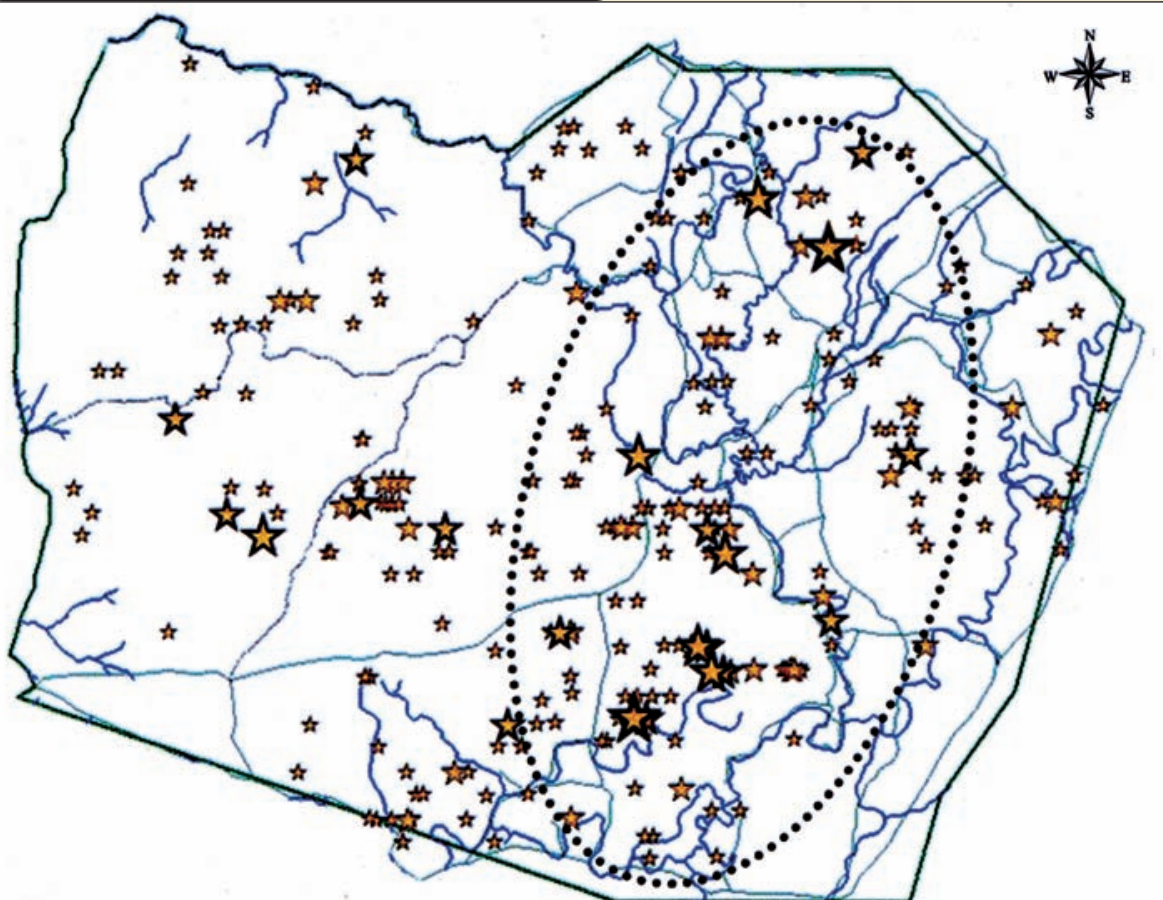

Limite Parc national

Hydrographie

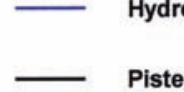

Piste
Vieilles carcasses

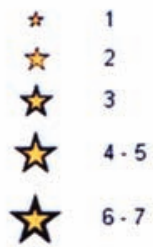

Échelle : $1 / 600000$

Figure 4.

Distribution des « vieilles » carcasses d'après les observations du comptage aérien d'avril-mai 2008.

Le parc national de Zakouma abrite près de $70 \%$ de la population d'éléphants estimée du Tchad et constitue donc un véritable sanctuaire pour cette espèce. Cette population, affaiblie à la suite des conflits armés des années 1980, s'est rapidement reconstituée dès les années 1990. Les effectifs obtenus au cours des différentes campagnes de comptages aériens, jusqu'en 2006, témoignent de l'efficacité des mesures d'aménagement et de protection mises en œuvre avec le soutien de partenaires internationaux. La structure d'âge et de sexe de la population, étudiée par DoLMIA (2004), est caractéristique d'une population jeune, en plein essor démographique (taux de reproduction de $2 \%$ ) avec très peu d'individus dont l'âge dépasse 50 ans. Cet auteur a montré que $17 \%$ des animaux disparaissaient par mort naturelle, $20 \%$ à la suite de causes indéterminées et $63 \%$ du fait du braconnage. La prédation intervient pour une part non négligeable dans la mortalité des animaux : en effet, VANHERLE (2006) a montré que la prédation par les lions se rapporte dans $26 \%$ des cas à de jeunes éléphants.

À Zakouma, le braconnage, comme dans de nombreux autres pays africains, a toujours constitué une menace sérieuse pour l'éléphant. Le lancement du projet Curess $^{1}$ en 2000, avec le soutien de l'Union européenne, avait permis de diminuer le braconnage dans les limites du parc, car la périphérie de l'aire protégée n'était pas prise en compte par les patrouilles de surveillance. La fin de la première phase de ce projet, en 2006, et l'attente du démarrage de la seconde phase ont conduit à une réduction des activités de surveillance pendant plusieurs mois. La mise en place du projet Curess II, en juillet 2007, n'a pas permis de stopper la recrudescence du braconnage. L'intervention d'un avion, mis à disposition du parc par la $W \mathrm{Cs}^{2}$ en début de la saison des pluies 2008, a cependant facilité la mise en œuvre d'un système de patrouille aérienne dissuasif qui s'est traduit par une légère réduction des massacres entre 2008 et 2009.

La chute drastique de la population d'éléphants sur une courte période, entre 2005 et 2009 , est très alarmante et laisse présager un avenir sombre pour la survie de l'espèce au Tchad : cela d'autant plus qu'elle coïncide avec la reprise du commerce de l'ivoire à l'échelle internationale.

\section{Références bibliographiques}

ARRANZ L., PFEFFER P., POILECOT P., 2007. Zakouma vingt ans après... Le Courrier de la Nature, 234 : 26-32.

BLANC J. J., BARNES R. F. W., CRAIG G. C., DUBLIN H. T., THOULESS C. R., DOUGLAS-HAMILTON I., HART J. A. 2007. African elephant status report 2007 : an update from the African elephant database. Gland, Suisse, Occasional Paper Series of the IUCN Species Survival Commission $n^{\circ} 33$.

BOUSQUET B., 1986. Projet de conservation du patrimoine naturel en zone Sud du Tchad. Seca/Ministère de l'Environnement et du Tourisme, Tchad.

BOUSQUET B., 1991. Parc national de Zakouma : résultats et inventaires de la faune. Projet « Réhabilitation et conservation du Parc national de Zakouma » ( $\left.n^{\circ} 6.800 .37 .51 .03\right)$. Seca/Ministère de l'Environnement et du Tourisme, Tchad, 27 p. + annexes.

DEJACE P., 1996. Suivi des éléphants dans le Sud du Tchad. Projet Fac 94 00300, 7-1996.

${ }^{1}$ Conservation et utilisation rationnelle des écosystèmes soudano-sahéliens, 2000 à 2006.

2 Wildlife Conservation Society. 
DEJACE P., GAUTIER L., BOUCHE P., 2000. Les populations de grands mammifères et d'autruches du Parc national de Zakouma au Tchad : statuts et tendances évolutives. La Terre et la Vie, $55: 305-320$.

DEPIERRE D., 1967. Les éléphants au Centre Tchad. Bois et Forêts des Tropiques, $113:$ 3-14.

DOLMIA N., 2004. Éléments d'écologie de la population d'éléphants du Parc national de Zakouma. Thèse en sciences de l'environnement, Engref, Montpellier, France, 335 p.

DOLMIA N. M., CALENGE C., MAIILARD D., PLANTON H., 2007. Preliminary observations of elephant (Loxodonta africana Blumenbach) movements and home range in Zakouma National Park, Chad. African Journal of Ecology, 45 : 594-598.

FAY J. M., DOLMIA N. M., BOULANODJI E., NDONINGA A., GUGGEMOS C., POILECOT P., 2005. Comptage aérien total de la grande faune du Parc national de Zakouma. Mee/Curess, Tchad, 35 p.

FAY J. M., N'GAKOUTOU E. B., TALOUA N., POILECOT P., NDONINGA A., 2006 a. Dénombrement aérien total des grands mammifères et de l'autruche du Parc national de Zakouma, Tchad. Mee/Dcfap/Curess, Tchad, $35 \mathrm{p}$.

FAY J. M., POILECOT P., N'GAKOUTOU E. B., TOMPKINS D., 2006 b. Dénombrement aérien total des grands mammifères dans le Parc national de Zakouma et sa zone d'influence. Mee/Dcfap/Curess, Tchad, 28 p.

FAY J. M., 2007. Les guerres de l'ivoire au Parc national de Zakouma. National Geographic, 90 : 24-53.

IUCN/SSC, 2007. African elephant specialist group. Gland, Suisse, lucn, $v i+276 \mathrm{p}$.

LOOMIS M., 2002. The elephants of Cameroon : mapping and analysing elephant migration. Site Web. North Carolina Zoological Park, www.nczooele track.org/ elephants/loomis_mapk/ (consulté en décembre 2009).

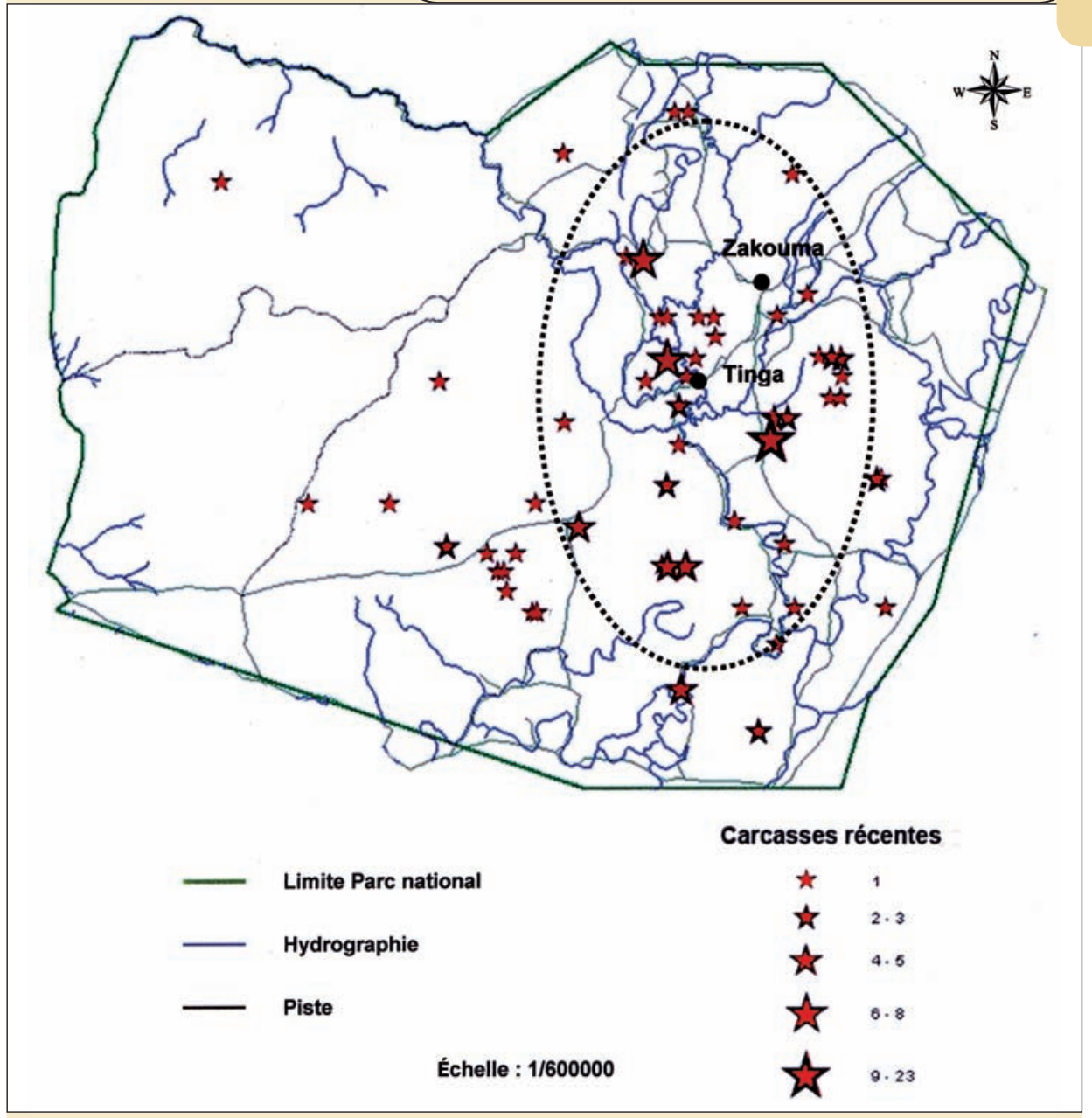

Figure 5.

Distribution des carcasses « récentes » d'après les observations du comptage aérien d'avril-mai 2008.

MACKIE C., 2002. Recensement aérien de la grande faune du Parc national de Zakouma. Mee/Dpfpn/Curess, Tchad, 29 p. + annexes.

PFEFFER P., THOMASSEY J.-P., 1986. Mission d'expertise faunique et d'évaluation potentielle du Parc national de Zakouma (République du Tchad). Paris, France, ministère des Relations extérieures, Coopération et Développement, 18 p.

POILECOT P., 2008. Comptage aérien des grands mammifères (avril 2008) et planification d'une stratégie de suivi écologique au Parc national de Zakouma (Tchad). Rapport final. Agrifor/Eco Consult/Gopa/Curess, 211 p.
POTGIETER D., TALOUA N., DJIMET B., FAY M., 2009. Dry season aerial total count, Zakouma national Park, Chad. Wcs/UE/Curess, $27 \mathrm{p}$.

TCHAMBA T. M., BAUER H., HUNIA A., DJOH A., NDIANG I., 1997. National elephant management Plan. Yaoundé, Cameroun, ministère de l'Environnement et des Forêts, Wwf.

VANHERLE N., 2006. Inventaire et suivi de la population de lions du Parc national de Zakouma. "Étude Lions Zakouma », Parc national de Zakouma (Sud-Est du Tchad). Rapport d'activité. Gland, Suisse, Iucn/Ssc. 